Bio - grafía. Escritos sobre la Biología y su Enseñanza. ISSN 2027-1034

Edición Extraordinaria. p.p. 764 - 770

Memorias del IX Encuentro Nacional de Experiencias en Enseñanza de la Biología y la Educación Ambiental. IV Congreso Nacional de Investigación en Enseñanza de la Biología.

\title{
ALGUNOS ANTECEDENTES DE INVESTIGACIÓN SOBRE TRANSPOSICIÓN DIDÁCTICA
}

\section{Angélica Carrillo Cajamarca ${ }^{1}$ Robinson Roa Acosta ${ }^{2}$}

\section{RESUMEN}

Este escrito presenta el resultado de indagar sobre algunas investigaciones realizadas bajo la categoría transposición didáctica, en el marco de una tesis de maestría que busca abordar la transposición didáctica del concepto de mezcla en profesoras de los grados de tercero, cuarto y quinto de primaria. En el rastreo se encuentra que la categoría en cuestión se ha puesto en juego para el caso de textos escolares de educación primaria y superior, la práctica de los profesores y procesos de enseñanza en ciencias de la salud, los cuales tienen como objetivo hacer esa transposición didáctica a los contenidos que son llevados al aula.

\section{PALABRAS CLAVE}

Transposición didáctica, textos escolares, práctica de profesores.

\section{ABSTRACT}

This paper presents the result of examine some researches carried out under the category of didactic transposition, within the framework of a master thesis that seeks to address the didactic transposition of the concept of mixture in teachers of the third, fourth and fifth grades of primary school. It is found that the category concerned has been brought in the case of school texts for primary and higher education, teachers' practice and teaching processes in health sciences; which are aimed to make the didactic transposition to the contents that are taken to the classroom.

\footnotetext{
${ }^{1}$ Estudiante de Maestría. Universidad pedagógica nacional. Licenciada en Química. Universidad Distrital Francisco José de Caldas. Profesora de la secretaría de educación de Bogotá. Colombia. eigna_cc@hotmail.com

${ }^{2}$ Doctor en Educación, Universidad Pedagógica Nacional. Magíster en Educación, Universidad Externado de Colombia. Licenciado en Biología. Universidad Pedagógica Nacional. Profesor de la Secretaria de Educación de Bogotá y profesor de cátedra Universidad Pedagógica Nacional. Bogotá. Colombia. robinsonroa@hotmail.com
} 
Bio - grafía. Escritos sobre la Biología y su Enseñanza. ISSN 2027-1034

Edición Extraordinaria. p.p. 764 - 770

Memorias del IX Encuentro Nacional de Experiencias en Enseñanza de la Biología y la

Educación Ambiental. IV Congreso Nacional de Investigación en Enseñanza de la Biología.

\section{KEY WORDS}

Didactic transposition, school texts, Teacher's practice.

\section{Con relación a la transposición didáctica}

Desde 1985, año en que Yves Chevallard retomó las ideas de Michel Verret (1975) para desarrollar el constructo de la transposición didáctica en el campo de las matemáticas, se amplió el espectro para abordar fenómenos relacionados con los procesos de enseñanzaaprendizaje, estableciendo relaciones entre la triada didáctica (el saber- el profesor y los estudiantes), inmersos en un sistema didáctico (entendido este, como las relaciones que se generan entre el profesor, los estudiantes y el conocimiento) y un contrato didáctico (proyecto compartido de enseñanza aprendizaje).

"...El concepto de transposición didáctica en tanto remite al paso del saber sabio al saber enseñado, y por lo tanto a la distancia eventual, obligatoria que los separa, da testimonio de ese cuestionamiento necesario, al tiempo que se convierte en su primera herramienta..." (Chevallard, 1991. p.16)

La transposición didáctica es un concepto abstracto, que requiere de procesos de análisis que lleven del objeto del saber al objeto a enseñar y de este al objeto de enseñanza; en este sentido se ha generado el interés por entender su aplicación.

\section{Transposición didáctica y los textos escolares}

Los textos escolares y universitarios (Solarte, 2006) son considerados recursos didácticos en los procesos de enseñanza aprendizaje, que poseen un enfoque y una perspectiva que contribuyen al proceso de construcción del conocimiento; poseen un diseño de actividades en los cuales se presentan los conceptos que pueden influir en la transformación del pensamiento.

Dentro del estudio de la categoría de transposición didáctica, se hizo una revisión de diferentes trabajos de investigación donde se encontró que el eje central de estos estudios era revisar la transposición didáctica que se hace en los libros de texto (universitarios o de básica secundaria). Para el caso de los textos universitarios se encuentran aquellos que eran empleados para enseñar los conceptos específicos del conocimiento de la química a profesores en formación inicial (modelo científico de LewisLangmuir, concepto de aromaticidad, estructura química del ADN, modelos moleculares de Walther Kossel, Gilbert Newton Lewis y Linus Carl Paulin,modelo semicuántico de Bohr); para el caso de los textos escolares, se evidencian los libros de química de grado décimo y undécimo usados por profesores para enseñar un determinado concepto ( modelo científico de Lewis-Langmuir, balanceo de ecuaciones, enlace químico, trabajo de Amadeo Avogadro). 


\title{
Bio - grafía. Escritos sobre la Biología y su Enseñanza. ISSN 2027-1034
}

\section{Edición Extraordinaria. p.p. 764 - 770}

\author{
Memorias del IX Encuentro Nacional de Experiencias en Enseñanza de la Biología y la \\ Educación Ambiental. IV Congreso Nacional de Investigación en Enseñanza de la \\ Biología.
}

Una de las características de estos estudios, es la necesidad de reflexionar sobre la naturaleza de la química que se hace objeto de enseñanza, para ello se analizan los textos tanto de nivel escolar como universitario. Se realiza la auscultación de la transposición didáctica de concepto especifico de la química, por ejemplo, para García (2004) el tema de interés era el modelo de la estructura química del ADN; Muñoz (2006) el balanceo de ecuaciones por oxido-reducción; De la Peña (2006) el concepto de enlace químico; Moreno (2009a) modelos moleculares Walter Kossel Gilbert Newton Lewis y Linus Carl Pauling; Moreno (2009b) modelo semicuántico de Bohr; Bonilla (2010) trabajo de Amadeo Avogadro; Herreño (2010) modelo científico de Lewis - Langmuir; Montaña y Piraban (2014) el concepto de aromaticidad.

Estos trabajos ponen de relieve la importancia y la influencia de los libros de textos escolares y universitarios, dado que en estos se evidencia la transposición didáctica de los conceptos; es así que los libros de texto presentan la forma como debe enseñarse química y la intencionalidad que persigue. Así también, los libros son resultado de la interpretación histórico- epistemológica de quienes los elaboran, por lo que entonces contienen visiones de ciencia.

Además, de acuerdo a estos trabajos de investigación, se evidencia la ausencia de la enseñanza de los aspectos histórico-epistemológicos; la dejación del proceso por el cual fue concebido ese conocimiento, se puede decir que existe una descontextualización al no considerar la formulación original del concepto. Los conceptos son presentados con posibles distorsiones, ambigüedades y omisiones de lo que planteaban originalmente los científicos.

De manera general, los trabajos de investigación revisados permiten concluir que la visión de ciencia que se presenta en los textos escolares y universitarios (empleados en los primeros semestres), es reduccionista con respecto a los conceptos, mostrando un conocimiento definitivo, acabado, cierto, absoluto, desconociendo el contexto de las instituciones donde se enseña, bajo una mirada "universalista", "positivista" de las ciencias de la naturaleza.

Estas investigaciones invitan a que los profesores se acerquen de manera reflexiva y crítica a los textos empleados para la enseñanza, pues de manera directa estos afectan su práctica pedagógica.

Por otra parte, Alfaro y Chavarría (2012) analizaron la transposición didáctica presente en la enseñanza y aprendizaje del conjunto de los números enteros en el nivel séptimo del sistema educativo costarricense; hicieron seguimiento a los docentes, estudiantes, libros de texto, y al sistema administrativo que impartía las directrices educativas; evidenciando la distancia existente entre cada uno de los saberes vinculados a la enseñanza y aprendizaje.

Alfaro y Chavarría (2012) encontraron que los libros empleados para enseñar el concepto de número entero, abordan cada uno de los contenidos propuestos por el currículo escolar, visualizan una perspectiva reduccionista de la enseñanza de la matemática (memorización y procesos algoritmos), y reflejan el problema de la ausencia de una 


\title{
Bio - grafía. Escritos sobre la Biología y su Enseñanza. ISSN 2027-1034
}

\section{Edición Extraordinaria. p.p. 764 - 770}

\author{
Memorias del IX Encuentro Nacional de Experiencias en Enseñanza de la Biología y la \\ Educación Ambiental. IV Congreso Nacional de Investigación en Enseñanza de la \\ Biología.
}

noosfera (según Chevallard) que regule el saber a enseñar y el saber enseñado, privada de intereses políticos. Se evidencia, además, el afán de abarcar la mayor cantidad de contenidos del saber sabio, incorporados estos al programa de enseñanza, sin cuestionar antes su pertinencia o bien, evaluar su factibilidad como objeto de enseñanza, cabe anotar que esta investigación es de corte cuantitativo a diferencia de las de química.

Por otra parte, Jiménez (2013) realiza una evaluación de uno de los criterios de la transposición didáctica en una unidad de conocimiento especializado "sistema inmune", su interés primordial radica en identificar el concepto de Sistema Inmune en el conjunto de la biología a través de los libros de texto que utilizan los estudiantes de un programa de Odontología y en relación con el concepto de sistema inmune que utilizan los biólogos. Plantea establecer relaciones entre lo que dicen los biólogos y lo que dicen los textos con respecto al concepto de interés, evaluando el criterio de transposición didáctica con el uso de términos cotidianos para aludir a nombres científicos y la incorporación o no de nombres científicos en los libros de texto de estudio.

Evidencia el autor que los conceptos tienen transformaciones mediante simplificaciones y distorsiones cuando pasan del texto científico al libro de texto, por tanto, es posible evidenciar los cambios que sufre un contenido desde una perspectiva semántico-cognitiva y pragmática.

Haciendo uso de las TIC's, Velandía (2013) planteó el diseño e implementación de un aula virtual bajo los principios de la transposición didáctica para la enseñanza de la química en estudiantes de ciencias de la salud, medicina, enfermería, nutrición y odontología. Se resalta que en su marco teórico presenta las grandes ideas de la transposición didáctica, sin embargo sus conclusiones apuntan más hacia las aulas virtuales dejando de lado el ejercicio de la transposición didáctica, al igual, destaca el papel actual de las tecnologías en los procesos educativos, en especial con instituciones de educación superior.

\section{Transposición didáctica y prácticas de los profesores}

El funcionamiento del sistema didáctico es muy diferente al sistema académico, lo cual hace que el estudio de la transposición didáctica sea de relevancia para apreciar los procesos de enseñanza, pues atañen directamente al profesor y la producción del conocimiento. Sin embargo, se encuentran pocos estudios sobre la transposición didáctica y las prácticas de los profesores, es decir, qué y cómo enseñan los contenidos.

Por tanto, continuando con la exploración de los trabajos de investigación que dan cuenta de la transposición didáctica desde el ejercicio de la práctica docente, se encuentra la de Díaz y Poblete (2009) quienes establecen una relación entre competencias y transposición. Buscan perfeccionar las competencias didácticas del profesor de matemática, dentro de sus conclusiones destacan que el diseño, implementación y puesta en práctica de este proyecto de investigación, así como su desarrollo, permitió capacitar en matemática a profesores con formación general básica, al igual que mejorar las competencias de los profesores logrando cambios en sus prácticas pedagógicas, 


\title{
Bio - grafía. Escritos sobre la Biología y su Enseñanza. ISSN 2027-1034
}

\section{Edición Extraordinaria. p.p. 764 - 770}

\author{
Memorias del IX Encuentro Nacional de Experiencias en Enseñanza de la Biología y la \\ Educación Ambiental. IV Congreso Nacional de Investigación en Enseñanza de la \\ Biología.
}

Sepúlveda (2011) realiza su estudio en la categoría de transposición didáctica de los contenidos matemáticos, analiza los códigos sociolingüísticos en matemáticas de profesores de colegios rurales. Se evidencia que la transposición didáctica realizada por los docentes es deficiente, presentando resistencia a ser retroalimentados en sus prácticas. La vigilancia epistemológica que hacen los profesores es insuficiente pues la mayoría de ellos desvirtúa el objeto matemático, otros en cambio muestran su apego a dicho objeto, al punto de confundirlo con el fin de la matemática educativa.

Patiño, Hincapié \& Rincón (2012) pretenden evidenciar en su trabajo, como se enseñan los saberes dentro de la universidad. Para ello, establecen ciertas categorías entre las cuales se encuentra la transposición didáctica, ya que se puede presentar diferentes maneras y sentidos del saber para un curso de comunicación y lenguaje mediáticos de la Licenciatura en Comunicación e Informática, puntualmente, los autores destacan que:

\begin{abstract}
"Una transposición no es propiamente pura ya que se puede presentar de diferentes maneras, Por tanto, la transposición se presenta a través de la enseñanza de los saberes universitarios por medio del maestro y se evidencia en el aprendizaje de los estudiantes para tener un proceso pleno y lleno de aciertos y desaciertos dependiendo de los resultados mismos..." (Patiño, Hincapié \& Rincón, 2012, p. 45).
\end{abstract}

Rodríguez (2015) plantea identificar la transposición didáctica en las clases de actuación de tercer semestre a partir de un módulo diseñado específicamente para el desarrollo de esta clase. Haciendo una transcripción de las sesiones observadas, concluye que el módulo empleado en clase cumple con los objetivos y metas de cada una, lo cual podría aludir una adecuada transposición didáctica.

Linares (2015) evidencia la transposición didáctica que realiza una profesora en la enseñanza de lengua castellana, siguiendo un módulo diseñado para primaria. Se resalta la importancia de reflexionar y reconocer como se configura ese conocimiento, la relación con la práctica pedagógica, la transformación del saber desde su producción a nivel disciplinar hasta su producción a nivel didáctico.

Después de este recorrido, es posible evidenciar que el estudio de la transposición didáctica se ha extendido a otros campos de conocimiento (artes, lenguaje y comunicaciones) distintos a la las matemáticas, ampliando el espectro de estudio, lo cual contribuye a la investigación en educación.

Por último, cabe destacar que en su mayoría, las investigaciones están centradas en auscultar la trasposición didáctica que realizan los textos tanto de secundaria como de universidad, que en realizar un análisis de esta en las prácticas docentes, dando la impresión que solamente fuese posible evidenciar elementos de la transposición didáctica en los libros y que son estos los que dirigen la práctica docente 
Bio - grafía. Escritos sobre la Biología y su Enseñanza. ISSN 2027-1034

Edición Extraordinaria. p.p. 764 - 770

Memorias del IX Encuentro Nacional de Experiencias en Enseñanza de la Biología y la Educación Ambiental. IV Congreso Nacional de Investigación en Enseñanza de la Biología.

\section{REFERENCIAS}

Alfaro, C. y Chavarría, J. (2012). La Transposición Didáctica: Un ejemplo en el sistema educativo costarricense. Uniciencia, 26 (1y2), 153-168. Recuperado de http://www.revistas.una.ac.cr/index.php/uniciencia/article/view/3870

Bonilla, K. (2010). Transposición didáctica del trabajo de Amadeo Avogadro (Tesis de Maestría). Universidad Pedagógica Nacional. Bogotá.

Chevallard, Y. (1991). La transposición didáctica. Del saber sabio al saber enseñado. Aique.

De la Peña, L. (2006). La transposición didáctica en el concepto de enlace químico (Tesis de Pregrado). Universidad Pedagógica Nacional. Bogotá.

Díaz, V. y Poblete, A. (2009). Competencias y transposición didáctica: Binomio para un efectivo perfeccionamiento en matemática. Investigación y Postgrado, 24(2), 77-107. Recuperado en 17 de julio de 2017, de http://www.scielo.org.ve/scielo.php?script=sci_arttext\&pid=S131600872009000200005\&lng=es\&tlng=es.

García, A. (2004). La transposición didáctica del modelo de la estructura química del ADN. Un análisis de textos de enseñanza universitaria. (Tesis de Maestría). Universidad Pedagógica Nacional. Bogotá.

Herreño, J., Gallego, R. y Pérez, R. (2010). Transposición didáctica del modelo científico de lewis-langmuir. Revista Eureka sobre Enseñanza y Divulgación de las Ciencias, Sin mes, 527-543

Jiménez, J. (2013). Evaluación de un criterio de transposición didáctica: El caso de la unidad de conocimiento especializado "sistema inmune". (Tesis de Maestría). Universidad Autónoma. Manizales.

Linares, A. (2015). Transposición didáctica: saberes disciplinares que fundamentan conceptualmente la configuración didáctica de una práctica de enseñanza de la lengua en educación básica primaria (Tesis de Maestría). Pontificia universidad Javeriana. Bogotá.

Montaña, J. y Pirabán, M. (2014). La transposición didáctica del concepto de aromaticidad en los libros de texto (Tesis de Maestría). Universidad Pedagógica Nacional. Bogotá.

Moreno, M. (2009 a). Transposición didáctica de los modelos moleculares Walter Kossel, Gillbert Newton Lewis y Linus Carl Pauling (Tesis de Maestría). Universidad Pedagógica Nacional. Bogotá.

Moreno, J. (2009 b). La transposición didáctica del modelo semicuántico de Bohr (Tesis de Maestría). Universidad Pedagógica Nacional. Bogotá. 
Bio - grafía. Escritos sobre la Biología y su Enseñanza. ISSN 2027-1034

Edición Extraordinaria. p.p. 764 - 770

Memorias del IX Encuentro Nacional de Experiencias en Enseñanza de la Biología y la

Educación Ambiental. IV Congreso Nacional de Investigación en Enseñanza de la Biología.

Muñoz, C. (2006). Transposición didáctica en el balanceo de ecuaciones por oxido reducción (Tesis de Pregrado). Universidad Pedagógica Nacional. Bogotá.

Patiño, J., Hincapié, L., y Rincón, S. (2012). Enseñar en la universidad. "Problematización, transposición didáctica y sentidos del saber, en el curso de comunicación y lenguajes mediáticos de la licenciatura en comunicación e informática educativas" (Tesis de Pregrado). Universidad tecnológica de Pereira. Pereira.

Rodríguez, G. (2015). La transposición didáctica como transformación de saberes (Tesis de Pregrado). Universidad Pedagógica Nacional. Bogotá.

Sepúlveda, K. (2011). Transposición didáctica de los contenidos matemáticos y Códigos socio lingüísticos profesores de escuelas rurales. XIII conferencia interamericana de educación matemática. CIAEM. Recife- Brasil.

Solarte, M. (2006). Los conceptos científicos presentados en los textos escolares: son consecuencia de la transposición didáctica. En: Revista Electrónica de la Red de Investigación Educativa [en línea]. Vol.1, No.4 (Enero-Junio de 2006)

Velandía, R. (2013). La transposición didáctica de conceptos de química y su relación con la enseñanza de las ciencias de la salud en el diseño de aula virtual "QUISAS" (química, salud y sinergias) (Tesis de Maestría). Universidad nacional de Colombia. Bogotá. 